\title{
Simultaneous delivery of two patent arterial duct coils via one venous sheath
}

\author{
D De Wolf, H Verhaaren, D Matthys
}

Department of Paediatric Cardiology, Children's Hospital UZ Gent, De Pintelaan 185,9000 Gent,

Belgium

Correspondence to: daniel.dewolf@rug.ac.be Accepted for publication 28 May 1997

\begin{abstract}
A female child, 10 months of age, weighing $7 \cdot 2 \mathrm{~kg}$, was catheterised for closure of a patent arterial duct. Aortography was performed in the lateral projection and the minimum diameter of the arterial duct was assessed by comparing it to the size of the catheter. The duct size was estimated between 3 and $3.5 \mathrm{~mm}$ at the narrowest point, therefore, it was decided to deliver two $5 \mathrm{~mm}$ patent arterial duct coils to avoid placement of an $8 \mathrm{~mm}$ coil in this small child. Similar operations were subsequently performed in two further children. Simultaneous delivery of two coils via a single long venous sheath is easy, fast, and safe. This simple and inexpensive procedure can reduce irradiation and anaesthesia time.
\end{abstract}

(Heart 1997;78:201-202)

Keywords: patent arterial duct; children; coil occlusion

Coil occlusion of a patent arterial duct with a diameter of less than $3.5 \mathrm{~mm}$ has proven to be a valuable alternative to surgical closure or closure with a double umbrella. ${ }^{1-6}$ It is not uncommon that multiple coils are necessary to achieve complete closure in moderate ducts with a diameter exceeding $2.5 \mathrm{~mm}$ at the narrowest point. ${ }^{156} \mathrm{We}$ describe a technique to deliver two coils simultaneously in one duct. This simple technique can be useful in ducts in which the probability of the use of multiple coils or extra large coils to achieve complete occlusion seems high. ${ }^{156}$

\section{Case report}

A female child, 10 months of age, weighing $7 \cdot 2 \mathrm{~kg}$, was catheterised for closure of a patent arterial duct. The procedure was performed under general anaesthesia. The right femoral vein was percutaneously cannulated with a $5 \mathrm{~F}$ sheath. The arterial duct was crossed with a 5F Gensini catheter (USCI, Bard, Galway, Ireland). Aortography was performed in the lateral projection and the minimum diameter of the arterial duct was assessed by comparing it to the size of the catheter. As the duct size was estimated between 3 and $3.5 \mathrm{~mm}$ at the narrowest point, we decided to deliver two
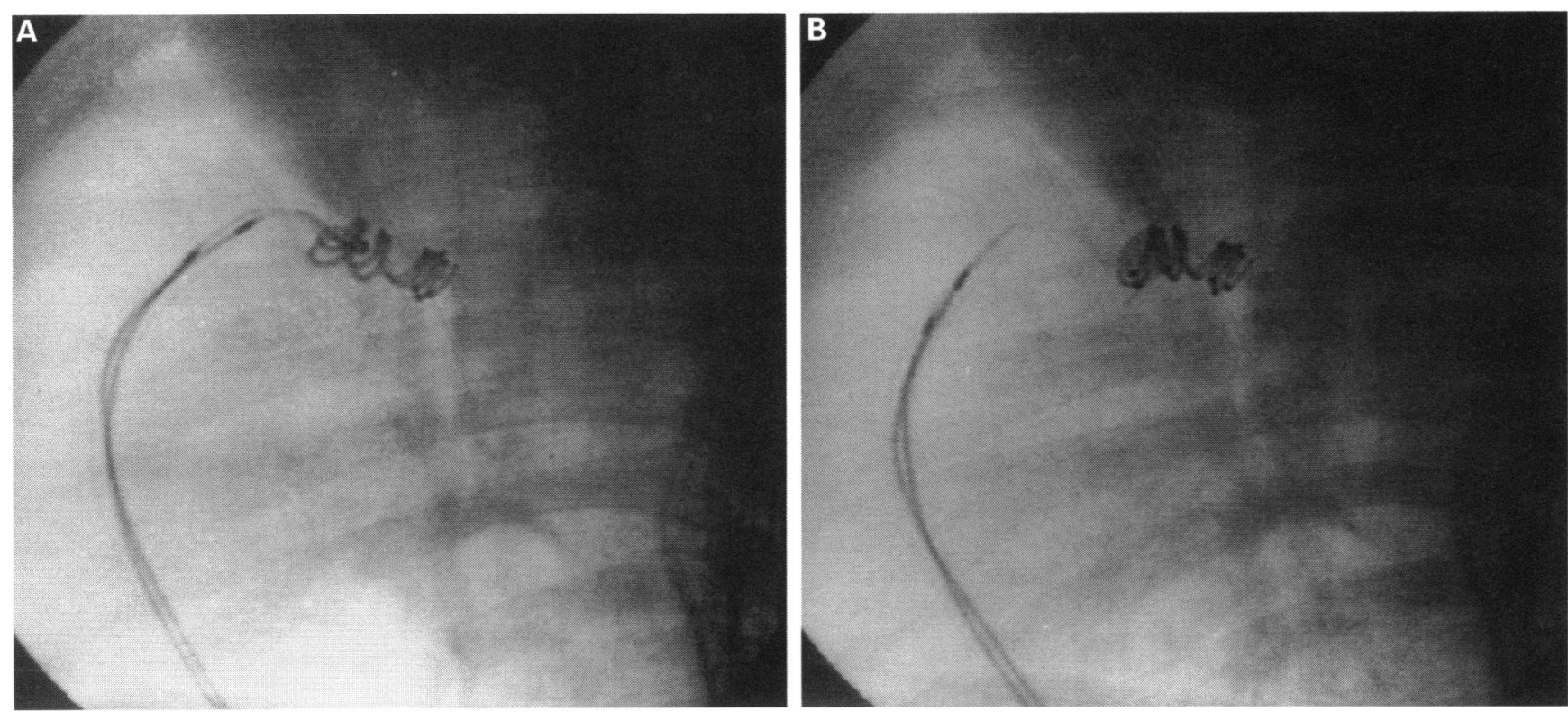

(A) Lateral view of the arterial duct, with the two coils still attached to the delivery wires in the venous sheath. (B) Lateral view of the package of the two coils after release, with the delivery wires withdrawn in the sheath. 
$5 \mathrm{~mm}$ Cook patent arterial duct coils (William Cook Europe, Bjaeverskov, Denmark) to avoid placement of an $8 \mathrm{~mm}$ coil in this small child.

We chose a single sheath approach. Manipulation of the coils within the sheath and unrestricted release of the coils through the system had already extensively been tested outside patients before this procedure. The Gensini catheter was exchanged for a $6 \mathrm{~F}$ Cook Mullins check-flo transeptal introducer set over a 0.025 inch Terumo guidewire positioned through the duct. Two $5 \mathrm{~mm}$ coils (five loops each) were introduced into the sheath and advanced together, with a slightly different position of half a loop. The first three loops of each coil were simultaneously pushed out of the sheath at the aortic side of the duct (fig A). Sheath and coils were withdrawn together and the remaining loops were simultaneously released at the pulmonic side (fig B).

After successful release complete occlusion of the duct and absence of stenosis of the left pulmonary artery were confirmed by echocardiography. The total procedure (induction of anaesthesia included) lasted 78 minutes with a total irradiation time of nine minutes.

\section{Further cases}

After this first patient we occluded patent arterial ducts following the same procedure in two further children. Two girls, one aged 6 months weighing $8 \mathrm{~kg}$, and one aged 15 months weighing $10 \mathrm{~kg}$, both presenting a $3 \mathrm{~mm}$ patent arterial duct, were successfully treated. A pair of $5 \mathrm{~mm}$ patent arterial duct coils was successfully delivered in each child, resulting in complete occlusion without any complication. The procedures lasted, respectively, 57 minutes with a total irradiation time of six minutes, and 62 minutes with a total irradiation time of seven minutes.

\section{Discussion}

Transcatheter closure of the patent arterial duct has become the method of choice in Europe. ${ }^{3}$ In some centres occlusion of the arterial duct, using detachable coils, has become a very safe and relatively inexpensive technique with a very high cumulative occlusion rate (up to $98 \%) .{ }^{1256}$ The technique is fairly straightforward in small ducts. The arterial duct with a narrow diameter, smaller than $2.5 \mathrm{~mm}$, can almost always be completely occluded with one coil with a diameter up to $5 \mathrm{~mm}$.

This is not the case for larger ducts. In these cases delivery of multiple or larger coils are needed to achieve complete occlusion. This makes the procedure more complex, necessitating longer procedural time, longer exposure to irradiation, and multiple angiograms, and increases the risk of dislocation of the original device. Multiple coils can be delivered in series or simultaneously in parallel via multiple delivery catheters. Recommendations for coil size differ and vary from the use of $8 \mathrm{~mm}$ coils in ducts of $3 \mathrm{~mm}$ and larger to the use of two simultaneously delivered $5 \mathrm{~mm}$ coils in ducts up to $3.5 \mathrm{~mm}$. $^{156}$

We found simultaneous delivery of two coils via a single long venous sheath easy, fast, and safe. It is a technique that can offer some advantages for the closure of the medium sized duct. It may reduce the use of single $8 \mathrm{~mm}$ coils for the closure of ducts no more than 3.5 $\mathrm{mm}$ in small children $(<8 \mathrm{~kg})$, decreasing the risk of left pulmonary artery stenosis. ${ }^{1}$ This simple and inexpensive $(£ 378$ of disposable material) procedure can reduce irradiation time (median times up to 18.9 and 40 minutes in series merely using multiple coils $)^{56}$ and anaesthesia time.

1 Uzun O, Hancock S, Parsons JM, Dickinson DF, Gibbs JL. Transcatheter occlusion of the arterial duct with Cook detachable coils: early experience. Heart 1996;76: 269-73.

2 Tometzki A, Chan K, De Giovanni J, Houston A, Martin $\mathrm{R}$, Redel D, et al. Total UK multi-centre experience with a novel arterial occlusion device (Duct Occlud pfm). Heart 1996;76:520-4.

3 Rigby ML. Closure of the arterial duct: past, present, and future [editorial]. Heart 1996;76:461-2.

4 Rosenthal E, Qureshi SA, Reidy J, Baker EJ, Tynan M. Evolving use of embolisation coils for occlusion of the arterial duct. Heart 1996;76:525-30.

5 Tometzki AJP, Arnold R, Peart I, Sreeram N, Abdulhamed JM, Godman MJ, et al. Transcatheter occlusion of the patent ductus arteriosus with Cook detachable coils. Heart 1996;76:531-5.

6 Hijazi ZM, Geggel RL. Transcatheter closure of large patent ductus arteriosus $(\geqslant 4 \mathrm{~mm})$ with multiple Gianturco coils: immediate and midterm results. Heart 1996;76:536-40 